"Facile modification of silica substrates provides a platform for direct-writing surface click chemistry". Oberhansl, S., Hirtz, M., Lagunas, A., Eritja, R., Martínez, E., Fuchs, H., Samitier, J. Small, 8(4), 541-545 (2012). doi: 10.1002/smll.201101875

\title{
Facile Modification of Silica Substrates Provides a Platform for Direct-Writing Surface Click Chemistry Sabine Oberhansl, ${ }^{a, b}$ Michael Hirtz, ${ }^{d}$ Anna Lagunas, ${ }^{a, b}$ Ramon Eritja, ${ }^{b, e, f}$ Elena Martinez, ${ }^{* a, b}$ Harald Fuchs, ${ }^{* d, g}$ and Josep Samitier, ${ }^{a, b, c}$ \\ ${ }^{a}$ Nanobioengineering Group , Institute for Bioengineering of Catalonia (IBEC) , C/Baldiri Reixac 15, 08028 Barcelona, Spain . E-mail: emartinez@ibecbarcelona.eu ${ }^{\mathrm{b}}$ Centro de Investigación Biomédica en Red en Bioingeniería Biomateriales y Nanomedicina (CIBER-BBN) C/María de Luna 11, Edificio CEEI, 50018 Zaragoza, Spain \\ 'Department of Electronics, University of Barcelona, C/Martí i Franquès 1, 08028 Barcelona, Spain \\ ${ }^{d}$ Institute of Nanotechnology (INT), Karlsruhe Nano Micro Facility (KNMF) , Karlsruhe Institute of Technology (KIT), Hermann-von-Helmholtz-Platz 1, 76344 Eggenstein-Leopoldshafen, Germany \\ ${ }^{\mathrm{e}}$ Institute for Research in Biomedicine (IRB Barcelona), C/Baldiri Reixac 10, 08028 Barcelona, Spain \\ Institute for Advanced Chemistry of Catalonia (IQAC), Spanish Research Council (CSIC), C/Jordi Girona 18, 08034 Barcelona, Spain \\ ${ }^{9}$ Westfälische Wilhelms-Universität and Center for Nanotechnology (CeNTech) \\ Heisenbergstrasse 11, 48149 Münster, Germany. E-mail: fuchsh@uni-muenster.de
}

As the "cream of the crop" of the newly introduced concept of click chemistry,[1] the Huisgen 1,3-dipolar cycloaddition of azides and alkynes presented a new strategy for the synthesis of triazoles.[2,3] In 2002, a significant breakthrough was reported independently by the groups of Meldal[4] and Sharpless,[5] who showed that the use of a copper(I) catalyst significantly increases the reaction efficiency and regioselectivity of the triazole formation. This so called copper (I)- catalyzed 1,3-diploar cycloaddition has been regarded as "the best click reaction to date"[6] and the "most efficient and widely used" one,[7] due to the mild reaction conditions and high tolerance of the functional groups. Recently, both Huisgen's and the copper (I)-catalyzed cycloaddition were applied for surface modification, as click reaction of monolayers was proven successfully on gold $[8,9,10]$ and silica[11,12] surfaces. They were considered very useful for the synthesis and modification of polymers, [1316] hydrogels,[17] or bioactive surfaces,[18] and for surface-patterning techniques like microcontact printing,[19-22] microarray fabrication,[23] or dip-pen nanolithography.[24-26] In order to perform the click reaction, the presence of two functional groups is necessary: an alkyne group and an azide group. Already existing strategies for click chemistry on surfaces generally focus on having the azide group on the reactive surface. Lin et al. looked at surface click reactions comparing the azide and alkyne groups immobilized on the substrate and found higher reaction yields when the azide group was on the surface.[23] The azide group is usually introduced 
by immobilizing a silane with a bromide group and transforming the bromide into an azide. However, this process is time consuming, with $49 \mathrm{~h}$ necessary for the reaction.[11,19] On the other hand, other groups have successfully performed surface click chemistry with a surface-bound alkyne group, $[12,19,20]$ which may be beneficial because of its higher stability.[24,27,28] An alkyne group can be introduced in an easy and wellestablished way, taking advantage of an already-existing epoxy group, which reacts upon ring-opening with an amine bearing an alkyne moiety.[29-32]

In this communication, we present a method to create a versatile and robust platform for surface click reactions on silicon oxide-based substrates, employing an uncomplicated and highly reproducible functionalization strategy which allows the direct writing of biomolecules. To this end, the surface is functionalized with a silane bearing an epoxy termination, which, in the second step, is subjected to a ring-opening reaction with propargylamine to give the alkyne group on the substrate (Figure 1). In contrast to the approach of Kuzmin et al.,[33] who have the more reactive epoxy group as a final group on the substrate, we preferred to have an alkyne group because of its stability.

Glass substrates were cleaned, silanized with (3-glyxidyloxypropyl)trimethoxysilane (GPTMS) and directly afterwards used for the next reaction step, in order to ensure the functional reliability of the epoxy group. The epoxy group was opened by adding propargylamine, resulting in an amino alcohol. Both functionalization steps can be considered clean since no side products are formed and no work-up is needed. On top of that, by using the epoxy group instead of a carboxyl moiety, no activation was necessary. In order to increase the stability of the platform for biological applications, the formation of an amino alcohol was preferred over the typical coupling via an amide group (peptide bond), because the latter is prone to being cleaved by proteases. All functionalization steps were checked by X-ray photoelectron spectroscopy (XPS) or time-of-flight secondary ion mass spectroscopy (ToF-SIMS), respectively (see Supporting Information (SI)).

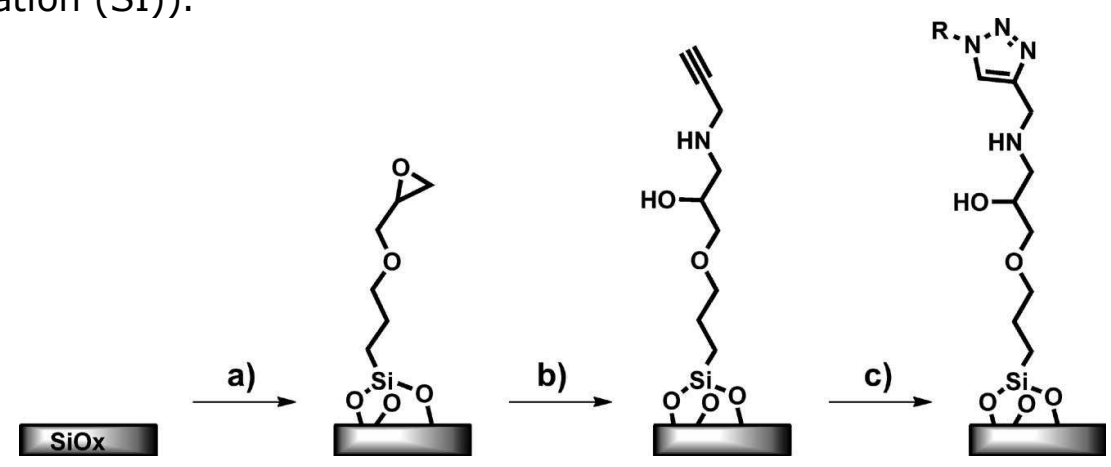

Figure 1. Functionalization strategy for silicon oxide-based substrates to enable surface click chemistry. a) Silanization step, including piranha cleaning and silanization with GPTMS. b) Ring-opening of the epoxy group with propargylamine to yield an amino alcohol. c) Surface click reaction of immobilized alkyne with incoming azide $\mathrm{R}-\mathrm{N}_{3}$ to yield the 1,2,3-triazole. 
In order to test the versatility of the substrates for click chemistry, three experiments were conducted. For the first one, a single-stranded oligonucleotide with an azide anchor was printed on an alkyne-modified glass slide by a robotic noncontact piezoelectric plotter (Nano-Plotter, GeSiM GmbH, Germany). The synthesis of the azido-oligonucleotide has been described previously.[34] Devaraj et al. already showed the immobilization of alkyne terminated oligos on an azide-terminated homogeneous self-assembled monolayer on gold via click reaction. [9] Spots of around $250 \mu \mathrm{m}$ in diameter (corresponding to drops of $0.4 \mathrm{~nL}$ in volume) were printed in an array configuration (Figure 2a) of solutions with and without catalyst (in this case $\mathrm{Cu}(\mathrm{I})$, tris[(1-benzyl-1H-1,2,3-triazol-4$\mathrm{yl}$ )methyl]amine (TBTA) and $N, N$-diisopropylethylamine (DIPEA) in dimethyl sulfoxide (DMSO)). Negative control spots were also included (Table 1).

After printing, the substrate was incubated for $12 \mathrm{~h}$ at room temperature to ensure the formation of the triazole ring between the surface-bound acetylene group and the incoming azide group from the oligonucleotidethis was also the case for solution B, which does not contain the catalystand subsequently hybridized with a complementary oligonucleotide strand bearing a fluorophore. The patterned area was visualized with fluorescence microscopy. Figure $2 \mathrm{a}$ shows the schematic set-up of the patterned area. The substrate modification itself did not give rise to autofluorescence. Figure $2 b$ shows fluorescent signal for solutions $B$ and $E$, which correspond to oligonucleotide with (E) and without $\mathrm{Cu}(\mathrm{I})(\mathrm{B})$, respectively. As expected, the solution with catalyst gives rise to a stronger fluorescent signal (E), since it is known that the presence of $\mathrm{Cu}(\mathrm{I})$ accelerates the cycloaddition reaction.[24,35] On the other hand, the oligonucleotide solution without catalyst ( $B$, corresponding to the original Huisgen's reaction) also gives rise to a fluorescent signal, though a weaker one, and it is important to know that the reaction will also take place without the $\mathrm{Cu}(\mathrm{I})$ catalyst because the presence of $\mathrm{Cu}(\mathrm{I})$ can be critical[20,36] and, over time, degradation of the oligonucleotide will occur. This problem has already been reported $[37,38]$ and can be reduced by using a $\mathrm{Cu}(\mathrm{I})$ binding ligand,[39] but not completely avoided. Negative controls did not give any signal after hybridization (solutions A, C, and D). The stability of the surface functionalization was tested by extensive washing before and after patterning. In a second experiment, a different patterning method was chosen, with the potential of making smaller features. Surface click chemistry has already been reported with dip-pen nanolithography (DPN),[24-26] so first we evaluated the feasibility of our substrates with DPN click functionalization. Therefore, a fluorescent ink (Alexa Fluor 555 azide) was deposited via DPN on the previously fabricated glass substrates. As a test pattern, we chose the lettering "KIT INT IBEC" to demonstrate the resolution and arbitrary shapes of lithography. The letter height is $3 \mu \mathrm{m}$, and the line width can be estimated from optical microscopy to be $300 \mathrm{~nm}$ or below (optical resolution limit of the microscope). Figure 3 a shows the resulting pattern and proves that the substrates are adequate for DPN click reactions. For the third experiment, a biotin modified with an azide group was attached covalently to a GPTMS/propargylamine-modified glass substrate via click chemistry. Having a biotin moiety on the substrate is especially interesting because it can be used for subsequent attachment of streptavidin and thus acts as a universal platform for the attachment of any molecule that can be biotinylated. $[40,41]$ The biotin-streptavidin pair is well known for its highly 
sensitive molecular biorecognition mechanism[42] and is often used for attaching delicate biomolecules to a substrate. The biotin pattern for this experiment was designed with future applications in mind and consists of an array of dots which can be used for cell experiments (unpublished data). DPN utilizes multitip arrays to enable high-throughput and large-area lithography; in this case a 1D 26-cantilever array with $35 \mu \mathrm{m}$ pitch was used. The pattern from a single cantilever consisted of $5 \times 5$ dots separated by $5 \mu \mathrm{m}$, yielding a $20 \mu \mathrm{m} \times 20 \mu \mathrm{m}$ square filled with dots. Twenty-six of these squares in the $x$-direction were written in parallel, then the array was moved $35 \mu \mathrm{m}$ in the $y$-direction and the next row of squares was generated. This procedure was repeated until 15 rows were created. After patterning, the surface was rinsed extensively and passivated against nonspecific protein adsorption. Finally, fluorescently labeled streptavidin was incubated on the surface in order to visualize the immobilized biotin. Figure $3 \mathrm{~b}$ shows the fluorescence image of Streptavidin-Cy3, which is specifically bound only to the areas patterned with immobilized biotin azide. A detailed description of the writing parameters, binding protocols, and a large-area overview of the fluorescent pattern is given in the SI.

a)

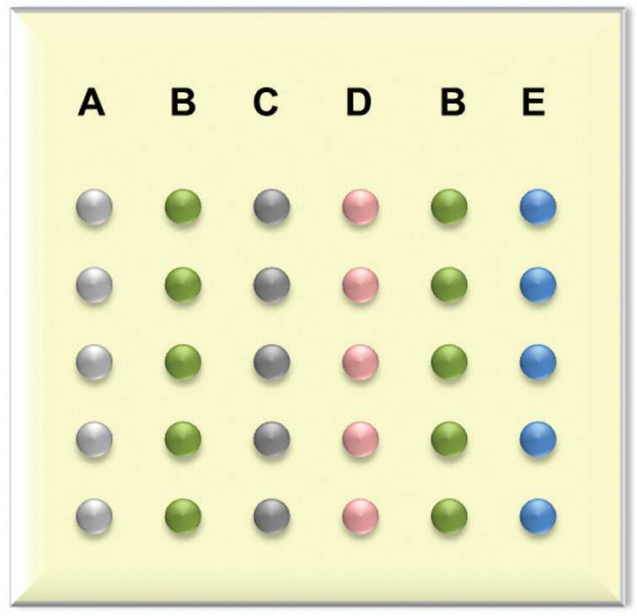

b)

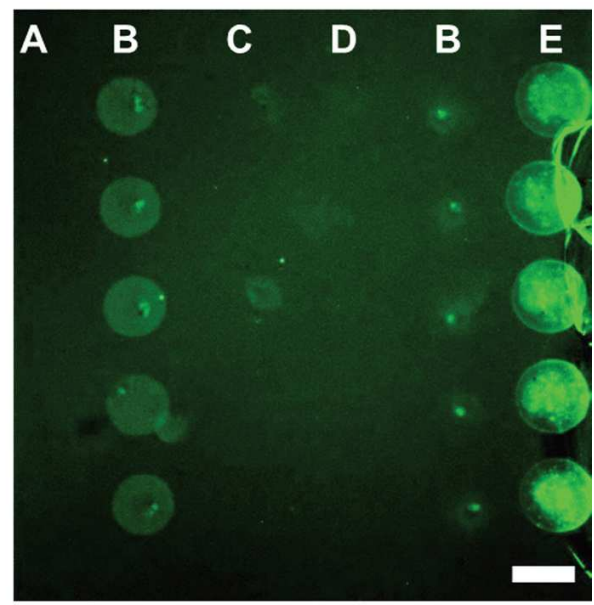

Figure 2. a) Schematic layout of the patterned area (for explanation see Table 1). b) Fluorescence microscope image of a functionalized glass substrate after patterning the azide-terminated oligonucleotide and incubation with a complementary strand bearing the dye Texas Red. Scale bar equals $200 \mu \mathrm{m}$.

Table 1. Solutions used for patterning with the Nanoplotter (negative controls are solutions $A, C$, and D).

Name

A

B

C

D

E
Solution
Solvent
CT oligo (azide group), NO catalyst Catalyst mixture
GA oligo (no azide group) + catalyst
CT oligo (azide group) + catalyst 

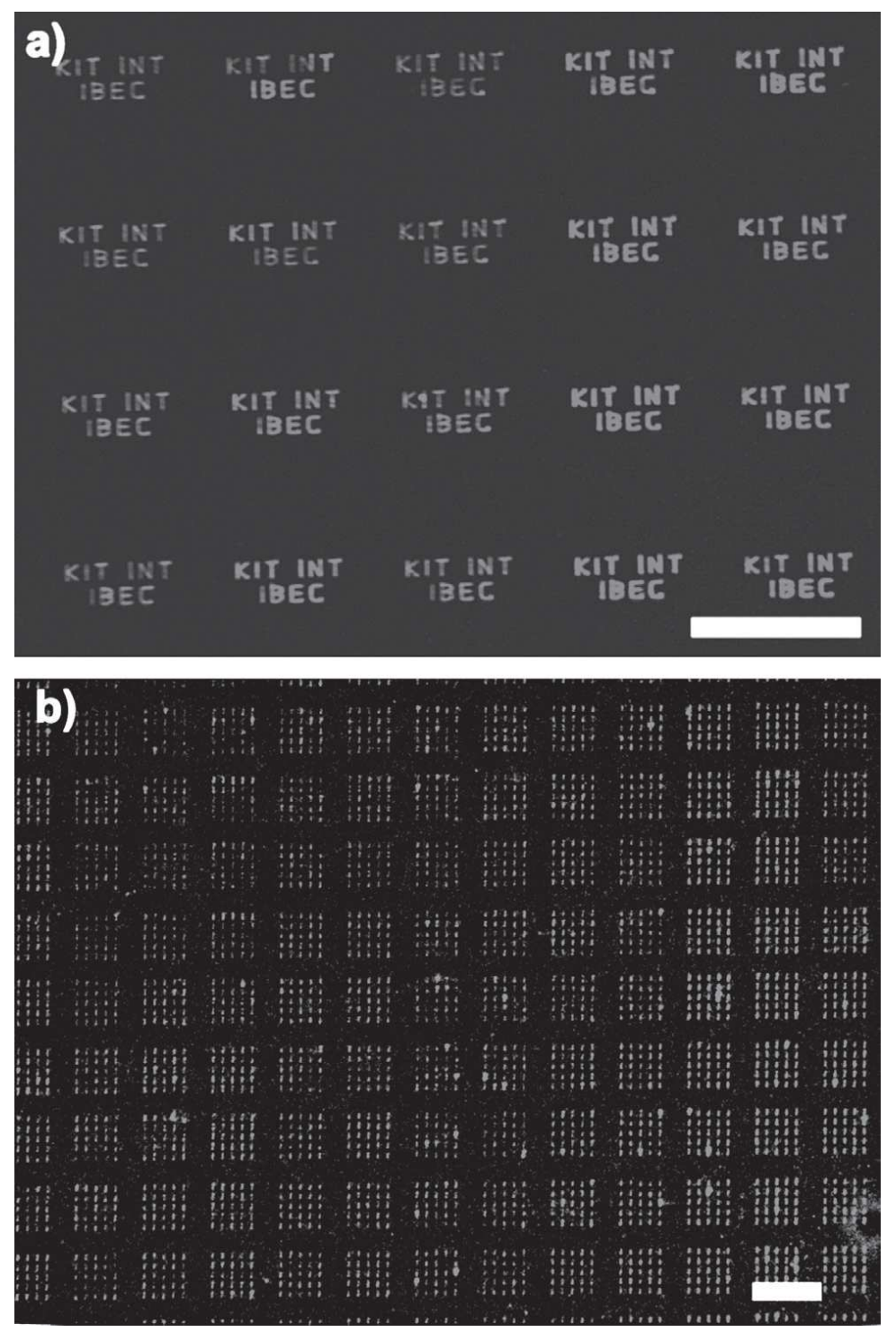

Figure 3. Fluorescence microscope images of a) an Alexa Fluor 555 azide ink structure deposited by dip-pen nanolithography and b) fluorescent StreptavidinCy3 bound on a biotin azide dot pattern generated by dip-pen nanolithography (image was corrected for background, see SI). Scale bars equal $35 \mu \mathrm{m}$.

In conclusion, we have shown a facile two-step functionalization strategy of silica substrates in order to perform surface click chemistry. Notably, no workup is needed and the surface functionalization, i.e., the alkyne group, is very stable, which allows for mass fabrication, subsequent storage and, most importantly, long term experiments without loss of functionality. Extensive washing steps were performed during the binding of oligonucleotides and biotin ink, proving an excellent stability and robustness of the covalently linked substrate functionalization and written patterns. Furthermore, the versatility of this direct patterning approach has been shown by using two direct-writing techniques at the micro-and nanoscale, 
as well as two different molecules, which were immobilized in a very stable manner. Both substrates (oligonucleotide- or biotin-modified) can be used for various applications ranging from biosensors to cell culture, since they are valuable platforms for attaching a broad range of molecules in a very specific and easy way.

\section{Experimental Section}

Functionalization of Silica Substrates: Glass slides were cleaned in piranha acid $\left(3: 1 \mathrm{v} / \mathrm{v}\right.$ solution of $\mathrm{H}_{2} \mathrm{SO}_{4}$ and $\mathrm{H}_{2} \mathrm{O}_{2}$ ) for 30 min, rinsed with copious amounts of MilliQ water and dried with pressurized air. Caution: piranha acid is a strong oxidizer and a strong acid. It should be handled with extreme care, as it reacts violently with most organic materials. The samples were then immersed in a solution of (3glycidyloxypropyl)trimethoxysilane (GPTMS) in toluene (1\% v/v) for $8 \mathrm{~h}$ at room temperature, afterwards rinsed with acetone, and dried with pressurized air. Immediately afterwards, the epoxy ring-opening was performed by immersing the substrates in a solution of propargylamine in acetonitrile $(2 \% \mathrm{v} / \mathrm{v})$ for $8 \mathrm{~h}$ at $45^{\circ} \mathrm{C}$. The samples were taken out of the solution, sonicated in ethanol for $5 \mathrm{~min}$, rinsed thoroughly with ethanol and MilliQ water and dried with pressurized air. If the samples were not used directly, they were stored at $4{ }^{\circ} \mathrm{C}$ under argon atmosphere.

Patterning of Oligonucleotides by Nanoplotter: Printing was carried out at $4{ }^{\circ} \mathrm{C}$ and $60 \%$ relative humidity with a robotic noncontact piezoelectric plotter (Nano-Plotter, GeSiM GmbH, Germany) using a so-called Nanotip. The plotted volume was $0.4 \mathrm{~nL}$ and the correct formation of the droplet was checked for each solution before patterning. Washing steps were done before and during patterning steps to minimize cross contamination. Oligonucleotide sequences and composition of the respective solutions for printing with the Nanoplotter are given in detail in the SI. After successful plotting, the surface was incubated overnight to ensure triazole formation and then rinsed with MilliQ and DMSO to remove unreacted oligonucleotides and catalyst (at this point, the sample was examined with time-of-flight secondary ion mass spectrometry to compare the surface functionalization). Subsequently, the substrate was incubated for $1 \mathrm{~h}$ at room temperature in $1 \%$ bovine serum albumin (BSA) in phosphate-buffered saline (PBS) to block unspecific adsorption, rinsed with PBS and incubated for $1 \mathrm{~h}$ at $36{ }^{\circ} \mathrm{C}$ with complementary oligonucleotide $A G(10 \mu \mathrm{m})$ in hybridization buffer $(10$ $\mathrm{mm}$ tris(hydroxymethyl)aminomethane (TRIS) base, $1 \mathrm{~mm}$ EDTA and $1 \mathrm{~m}$ $\mathrm{NaCl}$ ). Afterwards, the sample was rinsed with hybridization buffer and MilliQ and examined with fluorescence microscopy.

Patterning of Alexa Fluor 555 Azide and Biotin-Azide by Dip- Pen Nanolithography: Dip-pen nanolithography was done on a DPN 5000 system (Nanoink, Skokie, USA). A 1D cantilever array containing 26 Si3N4 was cleaned with oxygen plasma, $10 \mathrm{sccm}$ at 100 mTorr with $30 \mathrm{~W}$ for $5 \mathrm{~min}$. The cantilevers were subsequently immersed for $10 \mathrm{~min}$ in a solution of Alexa Fluor 555 azide (150 $\mu \mathrm{g} \mathrm{mL}^{-1}$ ) or biotin azide (PEG4 carboxamide-6- 


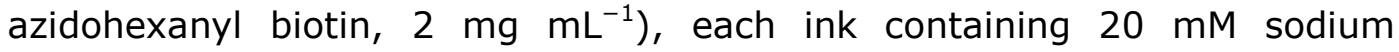
ascorbate and $10 \mathrm{mM} \mathrm{CuSO}_{4}$. After inking the cantilever arrays were blowdried with pressurized nitrogen. Writing of the Alexa Fluor 555 patterns was performed in feedback mode with a velocity of $1 \mu \mathrm{m} \mathrm{s}^{-1}$ at $74.8 \%$ relative humidity and $26.4^{\circ} \mathrm{C}$. The biotin azide dot patterns were generated with a dwell time of $2 \mathrm{~s}$ per dot at $74.0 \%$ relative humidity and $26.4{ }^{\circ} \mathrm{C}$. After the DPN process for the biotin pattern the sample was blocked with a PBS solution containing $0.5 \%$ BSA for 15 min, then washed with PBS three times and incubated for another 15 min with PBS containing 1 vol\% StreptavidinCy3 (Sigma-Aldrich $\mathrm{GmbH}$, Germany). After that, the sample was washed again three times with PBS, rinsed with MilliQ DI water and blow-dried with nitrogen before inspection with fluorescent microscopy. SI, Figure S1 shows a large area overview of the pattern after Streptavidin-Cy3 binding.

\section{Acknowledgements}

$\mathrm{SO}$ and $\mathrm{MH}$ contributed equally to this work. CIBER-BBN is an initiative funded by the VI National R\&D\&i Plan 2008-2011, Iniciativa Ingenio 2010, Consolider Program, CIBER Actions and financed by the Instituto de Salud Carlos III with assistance from the European Regional Development Fund. The Nanobioengineering group has support from the Commission for Universities and Research of the Department of Innovation, Universities, and Enterprise of the Generalitat de Catalunya (2009 SGR 505). This study was supported by "Fundación M. Botín", Santander, Spain. Part of the work was carried out with support of the European Community. We appreciate the support of the European Research Infrastructure EUMINAfab (funded under the FP7 specific program Capacities, Grant Agreement Number 226460) and its partner at the Karlsruhe Institute of Technology (KIT). This work was partly carried out with the support of the Karlsruhe Nano Micro Facility (KNMF, www.kmf.kit. edu), a Helmholtz Research Infrastructure at Karlsruhe Institute of Technology (KIT, www.kit.edu). We thank Margarita Alvira for the synthesis of the azido-oligonucleotide. This Communication is part of the Special Issue on Multilevel Molecular Assemblies: Structure, Dynamics, and Functions, featuring contributions from the Transregional Collaborative Research Center (TRR 61).

\section{REFERENCES}

[1] H. C. Kolb, M. G. Finn, K. B. Sharpless, Angew. Chem. Int. Ed. 2001, 40, 2004.

[2] R. Huisgen, Proc. Chem. Soc. 1961, 357.

[3] R. Huisgen, Angew. Chem. Int. Ed. 1963, 2, 565.

[4] C. W. Tornoe, C. Christensen, M. Meldal, J. Org. Chem. 2002, 67, 3057.

[5] V. V. Rostovtsev, L. G. Green, V. V. Fokin, K. B. Sharpless, Angew. Chem. Int Ed. 2002, 41, 2596.

[6] F. Himo, T. Lovell, R. Hilgraf, V. V. Rostovtsev, L. Noodleman, K. B. Sharpless, V. V. Fokin, J. Am. Chem. Soc. 2005, 127, 210. 
[7] S. Hohloch, C.-Y. Su, B. Sarkar, Eur. J. Inorg. Chem. 2011, 3067.

[8] J. P. Collman, N. K Devaraj, C. E. D. Chidsey, Langmuir 2004, 20, 1051.

[9] N. K. Devaraj, G. P. Miller, W. Ebina, B. Kakaradov, J. P. Collman, E. T. Kool, C. E. D. Chidsey, J. Am. Chem. Soc. 2005, 127, 8600.

[10] Y. Zhang, S. Luo, Y. Tang, L. Yu, K. Y. Hou, J. P. Cheng, X. Zeng, P. G. Wang, Anal. Chem. 2006, 78, 2001.

[11] T. Lummerstorfer, H. Hoffmann, J. Phys. Chem. B 2004, 108, 3963.

[12] X. L. Sun, C. L. Stabler, C. S Cazalis, E. L. Chaikof, Bioconjugate Chem. 2006, 17, 52.

[13] R. A. Evans, Aust. J. Chem. 2007, 60, 384.

[14] M. Bertoldo, G. Zampano, F. La Terra, V. Villari, V. Castelvetro, Biomacromolecules 2011, 12, 388.

[15] X. Huang, X.-J. Huang, A.-G. Yu, C. Wang, Z.-W. Dai, Z.-K. Xu, Macromol. Chem. Phys. 2011, 212, 272.

[16] X. Liu, H.-N. Zheng, Y.-Z. Ma, Q. Yan, S.-J. Xiao, J. Colloid Interface Sci. 2011, 358, 116.

[17] A. Koschella, M. Hartlieb, T. Heinze, Carbohydr. Polym. 2011, 86, 154. [18] P. Dutta, S. Sawoo, N. Ray, O. Bouloussa, A. Sarkar, Bioconjugate Chem. 2011, 22, 1202.

[19] H. Nandivada, H.-Y. Chen, L. Bondarenko, J. Lahann, Angew. Chem. Int. Ed. 2006, 45, 3360.

[20] D.I. Rozkiewicz, D. Janczewski, W. Verboom, B. J. Ravoo, D. N. Reinhoudt, Angew. Chem. Int. Ed. 2006, 45, 5292.

[21] F. A. Scaramuzzo, A. González-Campo, C.-C. Wu, A.H. Velders, V. Subramaniam, G. Doddi, P. Mencarelli, M. Barteri, P. Jonkheijm, J. Huskens, Chem. Commun. 2010, 46, 4193.

[22] J. J. Gassensmith, P. M. Erne, W. F. Paxton, C. Valente, J. F. Stoddart, Langmuir 2011, 27, 1341.

[23] P.-C. Lin, S.-H. Ueng, M.-C. Tseng, J.-L. Ko, K.-T. Huang, S.-C. Yu, A. K. Adak, Y.-J. Chen, C.-C. Lin, Angew. Chem. Int. Ed. 2006, 45, 4286.

[24] D. A. Long, K. Unal, R.C. Pratt, M. Malkoch, J. Frommer, Adv. Mater. 2007, 19, 4471 .

[25] W. F. Paxton, J. M. Spruell, J. F. Stoddart, J. Am. Chem. Soc. 2009, $131,6692$.

[26] H. Y. Chen, M. Hirtz, X. Deng, T. Laue, H. Fuchs, J. Lahan, J. Am. Chem. Soc. 2010, 132, 18023.

[27] "Azides are energy-rich molecules, ...] can be heat and shock sensitive and can decompose with little input of external energy." - taken from the website

http://www.stanford.edu/dept/EHS/prod/researchlab/lab/safety_sheets/08203.pdf, accessed July, 2011.

[28] S. Bräse, C. Gil, K. Knepper, V. Zimmermann, Angew. Chem. Int. Ed. 2005, 44, 5188.

[29] L. S. Penn, T. F. Hunter, Y. Lee, R. P. Quirk, Macromolecules 2000, 33, 1105.

[30] T. C. Hang, A. Guiseppi-Elie, Biosens. Bioelectron. 2004, 19, 1537.

[31] N. Y. Lee, B. H. Chung, Langmuir 2009, 25, 3861.

[32] E. Soto-Cantu, B. S. Lokitz, J. P. Hinestrosa, C. Deodhar, J. M. Messman, J. F. Ankner, S. M. Kilbey II, Langmuir 2011, 27, 5986.

[33] A. Kuzmin, A. Poloukhtine, M. A. Wolfert, V. V. Popik, Bioconjugate Chem. 2010, 21, 2076. 
[34] M. Alvira, R. Eritja, Chem. Biodivers. 2007, 4, 2789.

[35] J. F. Lutz, Angew. Chem. Int. Ed. 2007, 46, 1018.

[36] R. Manova, T. A. van Beek, H. Zuilhof, Angew. Chem. Int. Ed. 2011, $50,5428$.

[37] M. W. Kanan, M. M. Rozenman, K. Sakurai, T. M. Snyder, D. R. Lui, Nature 2004, 431, 545.

[38] R. Kumar, A. El-Sagheer, J. Tumpane, P. Lincoln, L. M. Wilhelmsson, T. Brown, J. Am. Chem. Soc. 2007, 129, 6859.

[39] T. R. Chan, R. Hilgraf, K. B. Sharpless, V. V. Fokin, Org. Lett. 2004, 6, 2853.

[40] A. Lagunas, J. Comelles, E. Martinez, J. Samitier, Langmuir 2010, 26, 14154.

[41] A. Lagunas, J. Comelles, E. Martinez, E. Prats-Alfonso, G. A. Acosta, F. Albericio, J. Samitier, Nanomedicine NBM 2011, DOI: 10.1016/j.nano.2011.08.001.

[42] E. A. Bayer, M. Wilchek, E. Skutelsky, FEBS Lett. 1976, 68, 240. 


\section{SUPPPLEMENTARY INFORMATION}

\section{Materials}

Glass Micro slides $75 \times 25 \mathrm{~mm}$ were purchased from Corning Inc. (NY, USA). AFM 1-D array $F$-type cantilevers (26 tips, $\mathrm{Si}_{3} \mathrm{~N}_{4}$ ) were bought from Nanoink Inc. (Skokie, Chicago, USA). Sulfuric acid $\left(\mathrm{H}_{2} \mathrm{SO}_{4}\right)$ 95-98\%, absolute ethanol, sodium chloride $(\mathrm{NaCl})$ and acetone were obtained from Panreac Química S.A.U. (Barcelona, Spain). 33\% w/v Hydrogen peroxide $\left(\mathrm{H}_{2} \mathrm{O}_{2}\right)$ was supplied by BASF (Barcelona, Spain). (3-glycidyloxypropyl)trimethoxysilane $\geq 98 \%$ (GPTMS), toluene $\geq 99.3 \%$, propargylamine $98 \%$, acetonitrile $\geq 99.9 \%$, dimethyl sulfoxide $\geq 99.5 \%$ (DMSO), Cu(I)I purum $\geq 99.5 \%, N, N$ diisopropylethylamine $\geq 99 \%$ (DIPEA), tris[(1-benzyl-1H-1,2,3-triazol-4yl)methyl]amine $97 \%$ (TBTA), ethylenediaminetetraacetic acid calcium disodium salt (EDTA), bovine serum albumin (BSA) and oligonucleotides (GA and $A G$ ) were purchased from Sigma-Aldrich Química S.A. (Madrid, Spain). ImmunoPure ${ }^{\circledR}$ Streptavidin was from Cultek S.L. (Madrid, Spain). Gold surfaces $(50 \mathrm{~nm}$ gold and $5 \mathrm{~nm}$ titanium on float glass) were obtained from NTB (Buchs, Switzerland). Dulbecco's phosphate buffered saline (PBS), Alexa Fluor ${ }^{\circledR} 555$ azide ink and PEG $_{4}$ carboxamide-6-azidohexanyl biotin ink were bought from Invitrogen $\mathrm{GmbH}$ (Germany). Tris(hydroxymethyl)aminomethane (TRIS) was obtained from Merck. Oligonucleotide CT was kindly provided by the group of Dr. Ramon Eritja at the Institute for Research in Biomedicine (IRB, Barcelona, Spain).

\section{Patterning of oligonucleotides by Nanoplotter}

Table S1. Oligonucleotide sequences used in experiments

\begin{tabular}{|l|l|l|}
\hline Entry & Name & Sequence $\left(5^{\prime}-3^{\prime}\right)$ \\
\hline 1 & CT & $N_{3}$-CTTCCTCCTCT \\
\hline 2 & GA & {$[$ 6Fluorescein]-GAAGGAGGAGA } \\
\hline 3 & AG & {$[$ Texas Red]-AGAGGAGGAAG } \\
\hline
\end{tabular}

The catalyst solution, containing CuI, TBTA and DIPEA was prepared separately in DMSO and added to the oligonucleotide directly before patterning. The oligonucleotides CT and GA were dissolved in MilliQ. The solvent for all mixtures and for plotting was DMSO : MilliQ $=1: 1$. 
Table S2. Solutions used for the Nanoplotter experiment

\begin{tabular}{|c|c|c|}
\hline Entry & Name & Concentration \\
\hline 1 & $\mathrm{E}(\mathrm{CT}+\mathrm{cat})$ & $11 \mu \mathrm{M} \mathrm{CT}, 1.1 \mathrm{mM} \mathrm{Cu}(\mathrm{I}) \mathrm{I}, 1.1 \mathrm{mM}$ TBTA, $1.3 \mathrm{mM}$ DIPEA \\
\hline 2 & $\mathrm{~B}(\mathrm{CT})$ & $11 \mu \mathrm{M} \mathrm{CT}$ \\
\hline 3 & $D(G A+c a t)$ & $11 \mu \mathrm{M}$ GA, $1.1 \mathrm{mM} \mathrm{Cu}(\mathrm{I}) \mathrm{I}, 1.1 \mathrm{mM}$ TBTA, $1.3 \mathrm{mM}$ DIPEA \\
\hline 4 & C (catalyst) & $1.1 \mathrm{mM} \mathrm{Cu}(\mathrm{I}) \mathrm{I}, 1.1 \mathrm{mM}$ TBTA, $1.3 \mathrm{mM}$ DIPEA \\
\hline 5 & A (solvent) & DMSO : MilliQ \\
\hline
\end{tabular}

\section{Patterning of Alexa Fluor ${ }^{\circledR} 555$ azide and biotin-azide by Dip-pen Nanolithography}

Out of focus stray light from alignment marks located right of the pattern in figures $3 b$ and S1was removed by ImageJ software (version 1.44p; Wayne Rasband, National Institutes of Health, USA) applying the "background subtract" option with the "sliding paraboloid" featurewith a rolling ball radius of 50 pixels.

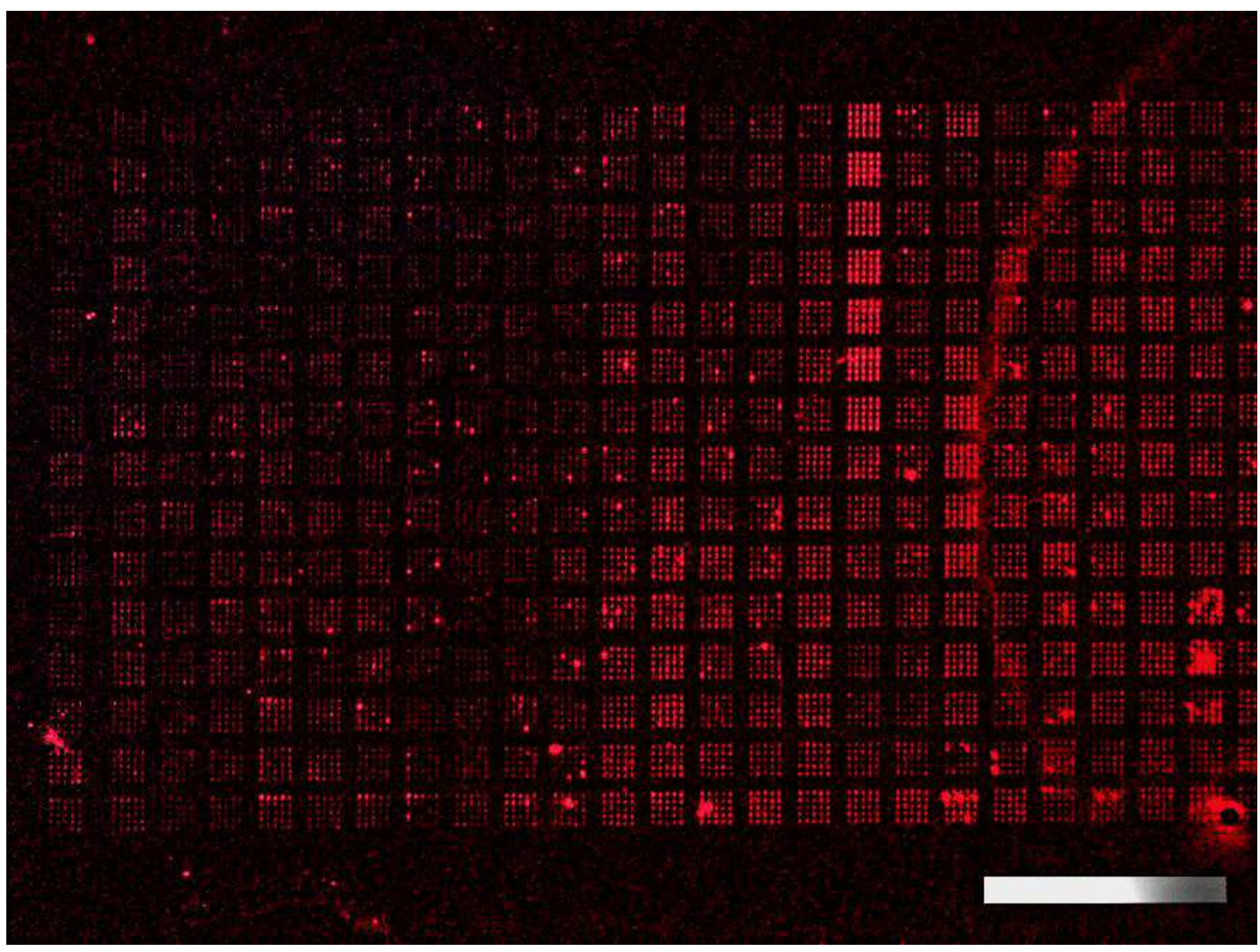

Figure S1. Fluorescent microscopy image of Streptavidin-Cy3 bound to a biotin azide pattern generated by DPN. Scale bar equals $175 \mu \mathrm{m}$. 


\section{Surface characterization via XPS and ToF-SIMS}

The two-step surface functionalization was monitored by XPS. ToF-SIMS experiments were performed as complementary measurements because it was not possible to analyze the phosphorus signature ( $P 2 p$ peak) with XPS measurements, as has been reported by Zhang et al.[1] Thus, the presence of DNA on the sample and negative controls was checked by ToFSIMS experiments. XPS measurements were performed using a PerkinElmer PHI 5500 Multitechnique System from Physical Electronics (Waltham, MA, USA) with a monochromatic X-ray source (Aluminum KR line of $1486.6 \mathrm{eV}$ energy and $350 \mathrm{~W}$ ) placed perpendicular to the analyzer axis and calibrated using the $3 \mathrm{~d} \mathrm{5/2}$ line of $\mathrm{Ag}$ with a full width at half-maximum (fwhm) of $0.8 \mathrm{eV}$. Core level scan spectra of carbon $1 \mathrm{~s}$, oxygen $1 \mathrm{~s}$, nitrogen $1 \mathrm{~s}$ and silicon $2 \mathrm{p}$ were recorded, whereas the analysis area was $\square 0.5 \mathrm{~mm} 2$ ). The resolution selected for the spectra was $187.5 \mathrm{eV}$ of pass energy and $0.8 \mathrm{eV} / \mathrm{step}$ for general spectra and $23.5 \mathrm{eV}$ of pass energy and $0.1 \mathrm{eV} /$ step for spectra of the different elements. All measurements were performed in an ultra-high vacuum (UHV) chamber with pressure levels between $7 \times 10-9$ and $3 \times 10-8$ mbar. When necessary, a low energy electron flood gun (0-3 eV) was used to discharge the samples. Peak fitting was performed using MultiPak V6.0A software from Physical Electronics Inc. (Chanhassen, MN, USA).

Figure S2 shows the results of the XPS analyses of high resolution spectra for $C$ 1s. Figure S2a shows the overlay of $C 1$ s signals of the different steps during the surface functionalization (see Figure 1, main article). The initial weak carbon peak is thought to be a result of the facile contamination of the activated surface after piranha treatment. It can be seen that the signal increases intensity when the GPTMS is anchored on the surface and at the same time, the maximum is shifted to higher energies and the signal becomes more complex. This can also be seen for the step of alkyne immobilization. Figure S2b shows the deconvolution of the glass surface where two peaks are obtained and are attributed to surface contamination as mentioned above. Figure S2c shows the deconvolution of the epoxy terminated surface where the initially obtained peaks from the glass surface were kept and the curve was fitted with 3 new peaks which correspond to $284.2 \mathrm{eV}$ (Si-C, purple), $285.8 \mathrm{eV}$ (CC/ C-H, green) and $287.7 \mathrm{eV}$ (C-O from ether groups, red). Figure S2d shows the deconvolution of the alkyne terminated surface where peaks were identified at $284.1 \mathrm{eV}$ (Si- C, purple) and $285.0 \mathrm{eV}$ (C-C/C-H/C $\square \mathrm{C}$, green), $286.3 \mathrm{eV}$ (C-O from ether and alcohol, red) and $288.8 \mathrm{eV}$ ( $\mathrm{C}-\mathrm{N}$, blue). The shift of the $\mathrm{C}-\mathrm{O}$ peak was expected since the newly created alcohol group has a lower binding energy (286 - 286.8 eV) compared to the ether group (286.3-288 eV). [2,3] 

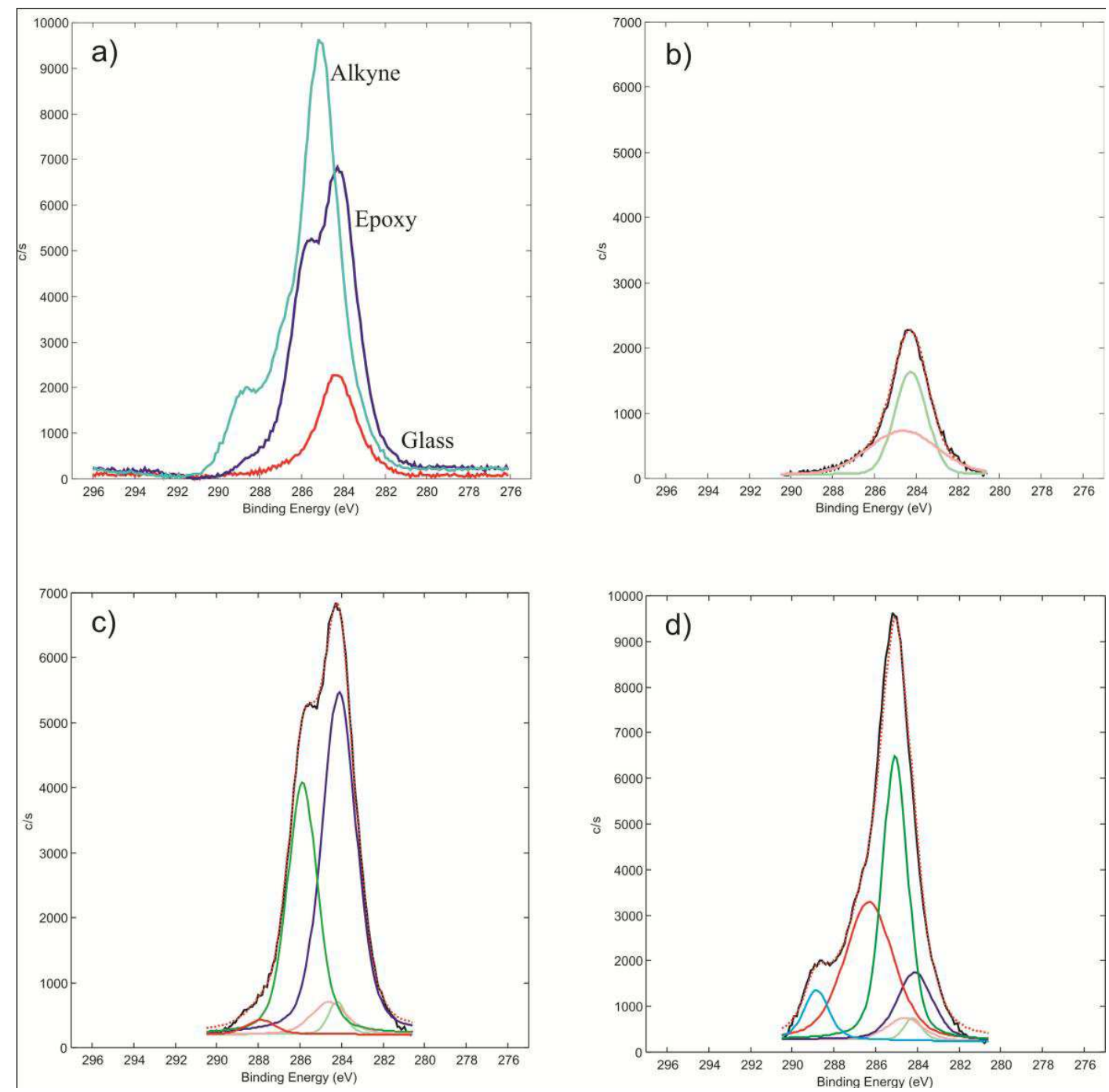

Figure S2. High resolution C 1s XPS spectra of (a) overlay of the three steps of the functionalization strategy and curve fits and deconvolution of (b) glass surface, (c) epoxy surface and (d) alkyne surface, to demonstrate the identified atomic components.

Figure S3 shows the high resolution XPS spectra of $\mathrm{N}$ 1s with Figure S3a being the overlay of all substrates, including the one where the click chemistry has been performed. It can be appreciated that on both, the glass and the epoxy surface, the $\mathrm{N}$ 1s signal is almost not existent. The alkyne surface shows an increase in signal and the deconvolution can be seen in Figure S3b, where the peak at $400.1 \mathrm{eV}$ (blue) is attributed to the amino group. [4] Figure S3c shows the deconvolution of the peak obtained for the click reaction surface, where 2 new components were identified as $\mathrm{N}-\mathrm{N}-\mathrm{C}$ at $400.1 \mathrm{eV}$ (green) and N-N-N at $401.5 \mathrm{eV}$ (pink). [5] 

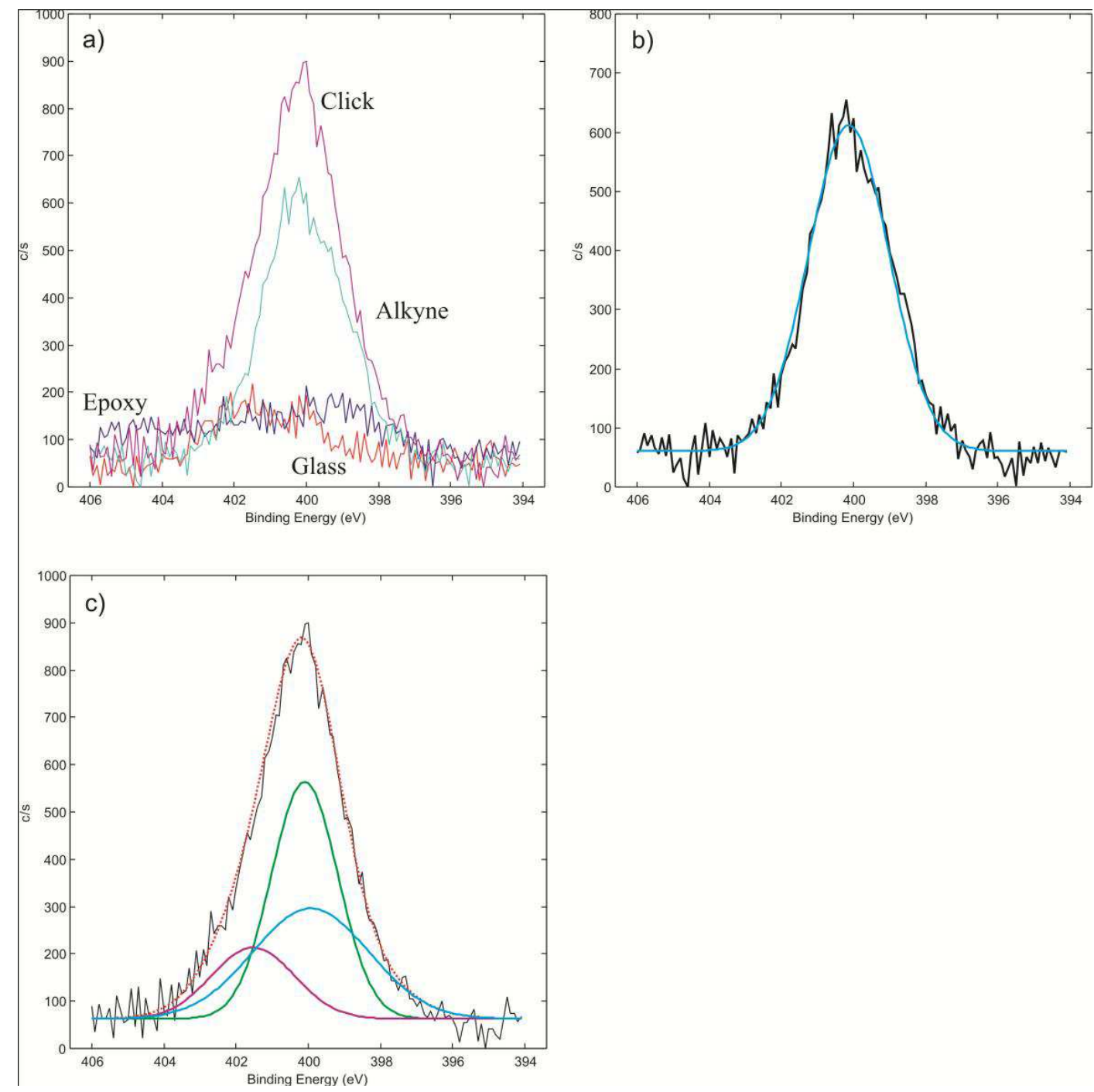

Figure S3. High resolution N 1s XPS spectra of (a) overlay of the three steps of the functionalization strategy and the click reaction substrate; Curve fit and deconvolution of (b) alkyne and (c) click reaction surface to demonstrate the identified atomic components.

Figure $\mathbf{S 4}$ shows the high resolution spectra of (a) $\mathrm{O} 1 \mathrm{~s}$ and (b) Si 2p. In both cases, the overlay of the three different steps of the surface functionalization (glass, epoxy and alkyne) can be seen. As expected, in both cases, the signal decreased slightly due to the covalent attachment of the mainly carbon and hydrogen containing molecules. 

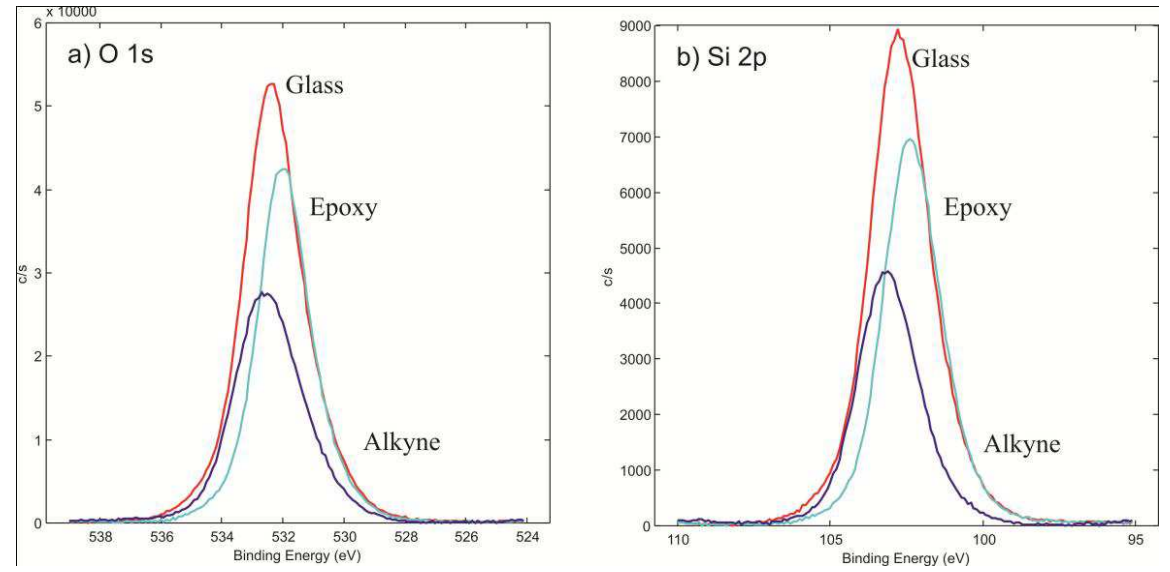

Figure S4. Overlay of the high resolution XPS spectra of (a) O 1s and (b) Si $2 p$ for the different steps of the functionalization strategy.

Measurement of the phosphorus signature for DNA was performed using a ToF-SIMS IV instrument (ION-ToF, Münster, Germany) operated at a pressure of $5 \times 10-9$ mbar. Samples were bombarded with a pulsed bismuth liquid metal ion source (Bi $3 p$ ), at $25 \mathrm{keV}$. The gun was operated with a 20 ns pulse width, $0.3 \mathrm{pA}$ pulsed ion current for a dosage lower than $5 \times 1011$ ions/cm2, well below the threshold level of 1 x 1013 ions/cm2 generally accepted for static SIMS conditions. Secondary ions were detected by a reflection time-of-flight analyzer, a multichannel plate (MCP) and time-todigital converter (TDC). Measurements were performed with a typical acquisition time of $20 \mathrm{~s}$, at a TDC time resolution of 200 ps. Charge neutralization was achieved with a low energy (20 eV) electron flood gun, thus no sample conductive coating was needed before the measurements. Secondary ions were extracted with $2 \mathrm{kV}$ voltage and were postaccelerated to $10 \mathrm{keV}$ kinetic energy just before hitting the detector. The maximum mass resolution, $R=m / \Delta m$, was around 8000 , where $m$ is the target ion mass and $\Delta \mathrm{m}$ is the resolved mass difference at peak half-width. Secondary ion spectra in negative mode were acquired from randomly rastered surface areas of $500 \mu \mathrm{m} \times 500 \mu \mathrm{m}$ along the slide.

Figure S5 shows the results of the ToF-SIMS experiments for the click reaction. Two substrates were incubated with the azide bearing CT oligo, one with catalyst (CT + cat) and one without catalyst (CT). Furthermore, two control surfaces were measured as well, namely bare glass and GA oligo without azide group but with catalyst (GA +cat). It is obvious that more oligonucleotide has been immobilized on the substrate for CT oligo with catalyst. Some CT oligo without catalyst has reacted with the alkyne group on the substrate but in a much lesser extent, as was expected from beforehand. There is still some GA oligo on the substrate, but this is rather due to unspecific adsorption than because of reaction, since the GA oligo lacks the azide group. More extensive washing and sonicating the surface will overcome this problem. 


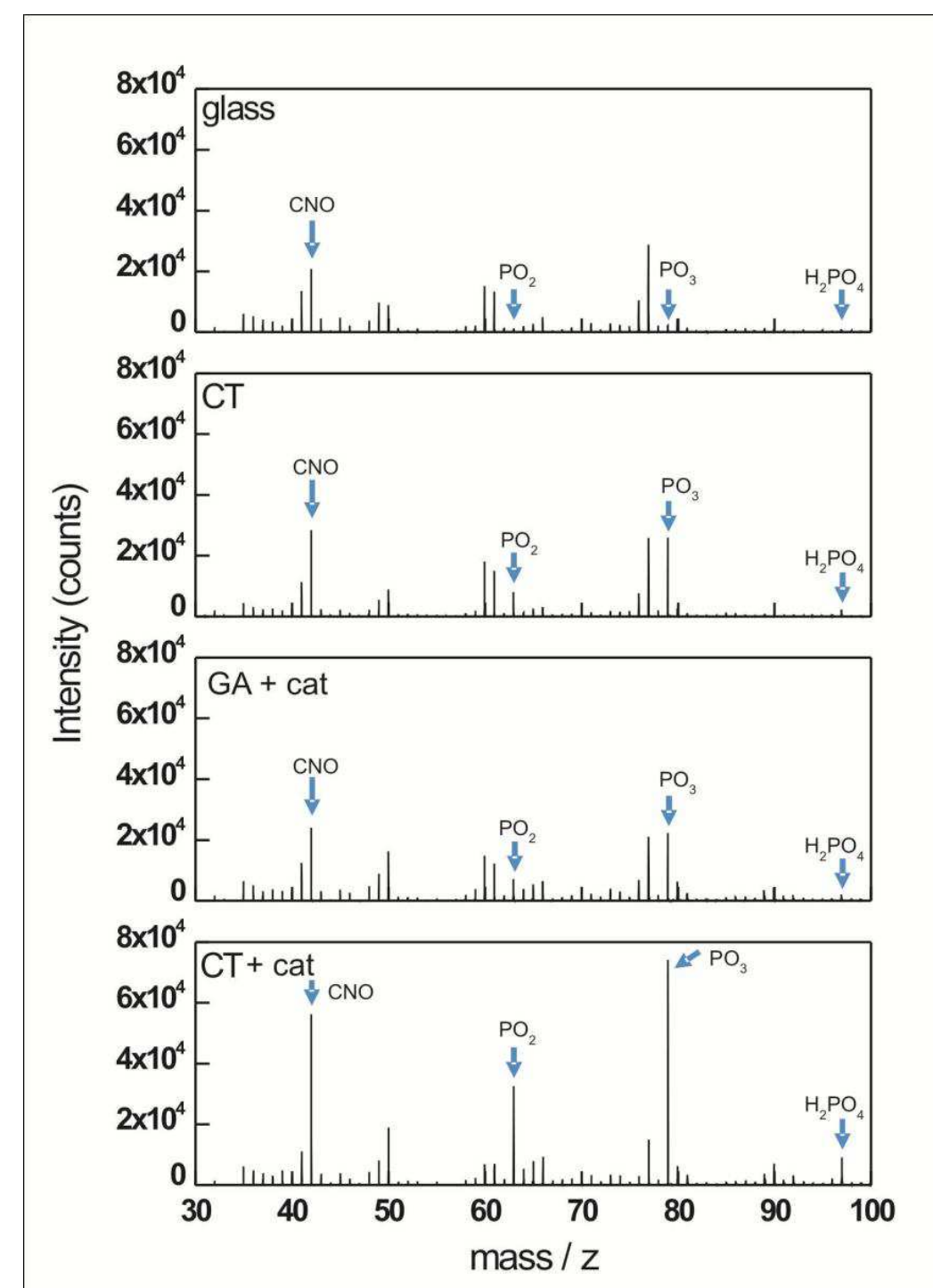

Figure S5. ToF-SIMS measurements of the surface click reaction. CT oligonucleotide was used with ( $\mathrm{Ct}+$ cat) and without catalyst (CT) and compared to negative controls: glass and GA oligo with cat (GA + cat), which lacks the azide group.

[1] X. Zhang, S. Kumar, J. Chen, A.V. Teplyakov, Surf. Sci. 2009, 603, 2445.

[2] L. Britcher, T.J. Barnes, H.J. Griesser, C.A. Prestidge, Langmuir 2008, 24, 7625.

[3] J.F. Moulder, W.F. Stickle, P.E. Sobol, K.D Bomben, in Handbook of Xray photoelectron spectroscopy: a reference book of standard spectra for identification and interpretation of XPS-data, (Eds: J.F. Moulder, J. Chastain), PHYSICAL ELECTRONICS, 1995, p.41 and 43, published by Perkin-Elmer Corporation, Minnesota, USA.

[4] T. Ramanathan, F.T. Fisher, R.S. Ruoff, L.C. Brinson, Chem. Mater. $2005,17,1290$.

[5] X. Liu, H.-N. Zheng, Y.-Z. Ma, Q. Yan, S.-J. Xiao, J. Colloid Interface Sci. $2011,358,116$. 